PROCEEDINGS OF THE

AMERICAN MATHEMATICAL SOCIETY

Volume 124, Number 5, May 1996

\title{
BOUNDARY VALUE ESTIMATES FOR HARMONIC FORMS
}

\author{
T. IWANIEC, M. MITREA, AND C. SCOTT
}

(Communicated by Albert Baernstein II)

\begin{abstract}
We prove a bound for the $L^{2}$-norm of harmonic forms in terms of certain $L^{p}$-norms of their normal and tangential components. In turn, this is used to show the $L^{2}$-norm equivalence of the normal and tangential components of harmonic forms on manifolds.
\end{abstract}

\section{INTRODUCTION AND STATEMENT OF RESUltS}

This note answers a question arising from discussion sparked between the authors during the Lexington, KY meeting of the AMS (\#890) on March 18-19, 1994. The problem we discuss here originates in the study of the Maxwell system on manifolds (cf. [JM94], [Mit94]).

Specifically, we shall prove the norm equivalence of the tangential and the normal components of a harmonic form $h$ of degree $1 \leq l \leq n-1$ (i.e. an $l$-form such that $d h=d^{*} h=0$ ) on an $n$-dimensional manifold $M$. For the duration of this note $M$ will be a smooth, oriented, Riemaniann, compact manifold with boundary $\partial M$ for which the deRham cohomology on $\mathrm{M}$ of degree $l$ and on $\partial M$ of degree $l-1$ are trivial (e.g. closed Euclidean balls). Also, the terms form and $C^{\infty}$-smooth form will be regarded as synonymous. This convention simplifies the citations from the literature and introduces no loss of generality since the results will be applied to harmonic forms which are, of course, $C^{\infty}$-smooth.

To explain how this relates to more classical results in Harmonic Analysis, assume that $(u, v)$ are two conjugate harmonic functions in the upper-half plane which decay at infinity. Then $u d x$ and $v d y$ are the tangential and the normal components of the harmonic form $h:=u d x+v d y$. Recalling that $H: u \mapsto v$ is the classical Hilbert transform, the next theorem can be regarded as a generalization of the boundedness of $H$ on $L^{2}\left(\partial \mathbb{R}_{+}^{2}\right)$.

Theorem 1.1. There exists $C>0$ such that for any harmonic form $h$ of degree $1 \leq l \leq n-1$ on $M$ we have

$$
C^{-1}\left\|h_{N}\right\|_{2, \partial M} \leq\left\|h_{T}\right\|_{2, \partial M} \leq C\left\|h_{N}\right\|_{2, \partial M},
$$

where $h_{T}, h_{N}$ are the tangential and normal components of $h$, respectively.

Received by the editors July 26, 1994 and, in revised form, October 17, 1994.

1991 Mathematics Subject Classification. Primary 31B25; Secondary 58G99.

Key words and phrases. Harmonic form, differential form, $\mathcal{L}^{p}$-norm.

The first author was partially supported by NSF grant DMS 9401104 .

(c)1996 American Mathematical Society 
This also extends well known results of Jerison, Kenig and Verchota concerning the $L^{2}$-norm equivalence of the normal and tangential derivative of harmonic functions in $\mathbb{R}^{n}$ (cf. also [Mit94]).

One of the major ingredients in the proof of this theorem is estimating the interior $L^{2}$-norm of a harmonic form in terms of its boundary normal and tangential components. Since this presents independent interest, we state here a more general version than the one we shall actually use.

Theorem 1.2. There exists $C>0$ such that for any harmonic form $h$ of degree $1 \leq l \leq n-1$ on $M, \frac{n-1}{n-2}<p<\infty$ and $n>2$, we have

$$
\|h\|_{2, M}^{2} \leq C\left\|h_{T}\right\|_{\tilde{p}, \partial M}\left\|h_{N}\right\|_{q, \partial M},
$$

where $\tilde{p}=\frac{(n-1) p}{(n-1)+p}$ and $q=\frac{p}{p-1}$.

As an illustration of this last result we also give

Corollary 1.3. For $n>2$ and any harmonic form $h$ on $M$ of degree $1 \leq l \leq n-1$,

$$
\|h\|_{2, M}^{2} \leq C\left\|h_{T}\right\|_{\frac{2(n-1)}{n}, \partial M}\left\|h_{N}\right\|_{\frac{2(n-1)}{n}, \partial M} .
$$

In particular,

$$
\|h\|_{2, M} \leq C_{n}\|h\|_{\frac{2(n-1)}{n}, \partial M} .
$$

The importance of these estimates resides in the fact that $1<2(n-1) / n<2$ when $2<n<\infty$. They are also relevant for the uniqueness part in the Dirichlet and Neumann problems for harmonic forms (i.e. determining a harmonic form $h$ on $M$ having a prescribed tangential and normal, respectively, component on $\partial M$ ).

Let us also mention that our results can be further refined when $M$ is the closure of a bounded Lipschitz domain in $\mathbb{R}^{n}$. Then $h$ can only be defined in the interior of $M$, with its nontangential maximal function in some appropriate $L^{p}(\partial M)$, and the boundary traces can be taken in the sense of nontangential convergence (cf. [Ken86]). In this case, all constants will depend only on the Lipschitz character of the domain.

\section{Preliminaries}

Letting $\wedge^{l} M$ denote the space of $l$-forms on $M$, we recall the exterior derivative

$$
d: \wedge^{l} M \rightarrow \wedge^{l+1} M
$$

as well as the Hodge star operator

$$
*: \wedge^{l} M \rightarrow \wedge^{n-l} M .
$$

For an arbitrary form $u$ on $M$, we define the tangential component of $u$ on $\partial M$ by

$$
u_{T}\left(X_{1}, \ldots, X_{n}\right)=u\left(\pi X_{1}, \ldots, \pi X_{n}\right)
$$

where $T M$ denotes the tangent bundle over $M$ and $\pi:\left.T M\right|_{\partial M} \rightarrow T(\partial M)$ denotes fibrewise orthogonal projection. We emphasize here that the vector fields $X_{1}, \ldots, X_{n}$ have values in $\left.T M\right|_{\partial M} \neq T(\partial M)$ and need only be defined on $\partial M$. However, when $X_{1}, \ldots, X_{n}$ take values only in $T(\partial M)$, we have

$$
u_{T}\left(X_{1}, \ldots, X_{n}\right)=u\left(X_{1}, \ldots, X_{n}\right) .
$$

The normal component of $u$ on $\partial M$ is, of course, given by $u_{N}:=u-u_{T}$ on $\partial M$. 
Denoting the Hodge codifferential operator $d^{*}=(-1)^{n(n-l)+1} * d *$, we recall the integration by parts formula

$$
\int_{M}\langle d u, v\rangle-\int_{M}\left\langle u, d^{*} v\right\rangle=\int_{\partial M} u_{T} \wedge * v_{N}
$$

Also, we will require a Gaffney type inequality. Several of these are exposed by Iwaniec, Scott and Stroffolini [ISS94] and we paraphrase one here.

Lemma 2.1. There exists a constant $C_{p}(1<p<\infty)$ so that for any l-form $u$ on $\partial M(l=0,1, \ldots, n-1)$, there is a closed l-form $u_{0}$ on $\partial M$ (i.e. $\left.d u_{0}=0\right)$ satisfying

$$
\left\|u-u_{0}\right\|_{p, \partial M}+\left\|d^{*}\left(u-u_{0}\right)\right\|_{p, \partial M} \leq C_{p}\|d u\|_{p, \partial M} .
$$

Also useful will be the following formulation of the Sobolev Imbedding Theorem (see Scott [Sco93]).

Lemma 2.2. There is a constant $C_{p}(1<p<n-1)$ so that

$$
\|u\|_{p^{*}, \partial M} \leq C_{p}\left(\|u\|_{p, \partial M}+\|d u\|_{p, \partial M}+\left\|d^{*} u\right\|_{p, \partial M}\right)
$$

where $u$ is any $l$-form $(l=0,1, \ldots, n-1)$ on $\partial M$ and $p^{*}=\frac{(n-1) p}{(n-1)-p}$.

For use in the proof of Theorem 1.2, we observe that the assumptions concerning deRham cohomologies from the introduction give

Lemma 2.3. Any closed form on $\partial M$ of degree $l$ with $0 \leq l \leq n-2$ may be extended to a closed form on $M$ of degree $l$.

Finally, we apply an integral identity used by several people in various contexts (for a general statement see [KW84], [EFM93], [JM94]) to readily obtain

Lemma 2.4. Let $\theta$ be a smooth vector field on $M$ and $\omega$ the 1-form dual to $\theta$. Also, let $i_{\theta}$ stand for the adjoint of the left exterior multiplication by $\omega$. Then, there is a constant $C>0$ so that

$$
\left.\left|\int_{\partial M}\langle\theta, N\rangle\right| h\right|^{2}-\left.2 \int_{\partial M}\left(i_{\theta} h\right)_{T} \wedge * h_{N}\left|\leq C \int_{M}\right| h\right|^{2} .
$$

for every harmonic form $h$.

\section{The PROOFs OF THE RESUlTS}

We first deal with Theorem 1.2. Let $h$ be a harmonic $l$-form on $M$ with $1 \leq l \leq$ $n-1$. By hypothesis, $h$ may be written as $h=d u$ for some $(l-1)$-form $u$. Notice that we may subtract from $u$ a closed form $v$ on $M$ without altering the validity of $h=d(u-v)$. Let $\frac{1}{p}+\frac{1}{q}=1$ and consider the estimate

$$
\begin{aligned}
\|h\|_{2, M}^{2} & =\int_{M} h \wedge * h \\
& =\int_{M} d(u-v) \wedge * h \\
& =\int_{\partial M}(u-v)_{T} \wedge * h_{N} \quad(\text { by } 2.1) \\
& \leq\left\|u_{T}-v_{T}\right\|_{p, \partial M}\left\|h_{N}\right\|_{q, \partial M} \quad(\text { Hölder }) \\
& \leq C_{p}\left(\left\|u_{T}-v_{T}\right\|_{\tilde{p}, \partial M}+\left\|d u_{T}\right\|_{\tilde{p}, \partial M}+\left\|d^{*}\left(u_{T}-v_{T}\right)\right\|_{\tilde{p}, \partial M}\right)\left\|h_{N}\right\|_{q, \partial M} .
\end{aligned}
$$


The last inequality follows by Lemma 2.2 since the hypothesis $\frac{n-1}{n-2}<p<\infty$ ensures that $1<\tilde{p}<n-1$. At this point, we exercise the freedom to choose $v$ on $M$ so that the estimate in Lemma 2.1 holds. Since this is possible thanks to Lemma 2.3, we may now continue our estimates with

$$
\begin{aligned}
\|h\|_{2, M}^{2} & \leq C\left\|d u_{T}\right\|_{\tilde{p}, \partial M}\left\|h_{N}\right\|_{q, \partial M} \\
& =C\left\|h_{T}\right\|_{\tilde{p}, \partial M}\left\|h_{N}\right\|_{q, \partial M} .
\end{aligned}
$$

This concludes the proof of the Theorem 1.2.

Note that, without invoking Lemma 2.1 and Lemma 2.2, the above reasoning also gives the rougher estimate

$$
\|h\|_{2, M}^{2} \leq C\left\|h_{T}\right\|_{2, \partial M}\left\|h_{N}\right\|_{2, \partial M}
$$

As for Corollary 1.3, we shall choose a suitable $p$. Solving $q=\tilde{p}$ gives

$$
1<q=\tilde{p}=\frac{2(n-1)}{n}<2 \leq n-1
$$

when $n \geq 3$, as desired. The last part of the corollary follows simply from the observation that $\left|h_{T}\right| \leq|h|$ and $\left|h_{N}\right| \leq|h|$.

Finally, turning our attention to the proof of Theorem 1.1, we first notice that one can construct a smooth vector field $\theta$ on $M$ so that $0<C \leq\langle\theta, N\rangle$ on $\partial M$. With this at hand, (2.2) and (3.1) yield

$$
\begin{aligned}
\|h\|_{2, \partial M}^{2} & \leq C\|h\|_{2, \partial M}\left\|h_{N}\right\|_{2, \partial M}+C\|h\|_{2, M}^{2} \\
& \leq C\|h\|_{2, \partial M}\left\|h_{N}\right\|_{2, \partial M}+C\|h\|_{2, \partial M}\left\|h_{N}\right\|_{2, \partial M} \\
& \leq \epsilon C\|h\|_{2, \partial M}^{2}+\frac{C}{\epsilon}\left\|h_{N}\right\|_{2, \partial M}^{2} .
\end{aligned}
$$

Choosing $\epsilon$ small enough and absorbing the small term into the left hand side, we get

$$
\|h\|_{2, \partial M} \leq C\left\|h_{N}\right\|_{2, \partial M}
$$

Using this and Hodge duality, we also obtain

$$
\|h\|_{2, \partial M} \leq C\left\|h_{T}\right\|_{2, \partial M}
$$

The proof of Theorem 1.1 is therefore complete.

\section{REFERENCES}

[EFM93] J.F. Escobar, A. Freire, and M. Min-Oo, Vanishing Theorems in Positive Curvature, Indiana Univ. Math. J. 42 (1993), 1545-1554. MR 95c:58173

[ISS94] T. Iwaniec, C. Scott, and B. Stroffolini, Nonlinear Potential Theory on Manifolds, (preprint) (1994).

[JM94] B. Jawerth and M. Mitrea, Higher Dimensional Scattering Theory on $C^{1}$ and Lipschitz Domains, (preprint) (1994).

[Ken86] C.E. Kenig, Elliptic Boundary Value Problems on Lipschitz Domains, Beijing Lectures in Harmonic Analysis, Annals of Math. Studies 112 (1986), 131-183. MR 88a:35066

[KW84] H. Karcher and J. Wood, Non-existence Results and Growth Properties for Harmonic Maps and Forms, J. Reine Angew. Math. 353 (1984), 165-180. MR 86g:58039 
[Mit94] M. Mitrea, Electromagnetic Scattering Theory on Nonsmooth Domains, (preprint) (1994). CMP 95:05

[Sco93] C. Scott, $L^{p}$ Theory of Differential Forms on Manifolds, Trans. Amer. Math. Soc. 347 (1995), 2075-2096. MR 95i:58009

(T. Iwaniec) Department of Mathematics, Syracuse University, Syracuse, New York 13244

E-mail address, T. Iwaniec: tiwaniec@mailbox.syr.edu

(M. Mitrea) School of Mathematics, University of Minnesota, 127 Vincent Hall, 206 Church Street S.E., Minneapolis, Minnesota 55455

E-mail address, M. Mitrea: mitrea@math.umn.edu

(C. Scott) Department of Mathematics, University of Wisconsin, 334 Sundquist Hall, SUPERIOR, WISCONSIN 54880

E-mail address, C. Scott: cscott@wpo.uwsuper.edu 